\title{
DENSIDAD POBLACIONAL Y DAÑOS OCASIONADOS POR LA ARDILLA Sciurus aureogaster: IMPLICACIONES PARA LA CONSERVACIÓN DE LOS VIVEROS DE COYOACÁN, MÉXICO
}

\author{
PATRICIA MORA-ASCENCIO, ÁNGELES MENDOZA-DURÁN \\ Y CUAUHTÉMOC CHÁVEZ
}

Instituto de Ecología, Universidad Nacional Autónoma de México. Aparto Postal 70-275, México,D.F.04510 MEXICO. correo electrónico: cchavez@ecologia.unam.mx

\begin{abstract}
Resumen: La ardilla roja mexicana (Sciurus aureogaster), aunque es una especie nativa del centro de México, fue introducida en los Viveros de Coyoacán a mediados del siglo XX y desde entonces ha ido habituándose a la presencia humana. En este trabajo estimamos la densidad poblacional de ardillas grises en los Viveros de Coyoacán, describimos sus patrones de actividad a lo largo de un año y cuantificamos los daños que producen en los árboles del parque. Para estimar la densidad poblacional, se realizaron conteos visuales por transecto lineal y por cuadrantes. Los patrones de actividad fueron evaluados en los transectos lineales. El daño fue evaluado según la causa y la especie de árbol, en 12 parcelas de $400 \mathrm{~m}^{2}$, y 13 puntos sobre un trayecto escogido al azar. Encontramos densidades poblacionales relativamente altas, en promedio de 5.9 a 6.5 inds./ha según los métodos de transecto y por cuadrantes, respectivamente. Con estos datos, estimamos entre 254 y 270 ardillas en todo el parque. Presentan dos picos de actividad, uno al amanecer y otro antes del anochecer. Poco más de la mitad (52\%) de los árboles muestreados (346) presentaron algún tipo de daño; de éstos el 80 \% fueron dañados por vandalismo y por poda, y el $20 \%$ restante por ardillas. Estos resultados revelan que es importante que se realicen campañas para no suplementar alimento a las ardillas y/o a otras especies y que exista un programa de capacitación para realizar una correcta poda de los árboles y con ello evitar daños mayores.
\end{abstract}

Palabras clave: Ardillas, densidad poblacional, patrones de actividad, Viveros de Coyoacán, Distrito Federal.

Abstract: The Mexican red squirrel (Sciurus aureogaster), but is a species native to central
Mexico, was introduced in the Viveros de Coyoacan mid twentieth century and has since been
habituated to human presence. In this paper we estimate the density of gray squirrels in the
Viveros de Coyoacan, we describe patterns of activity over a year and quantify the damage
occurring in the park trees. To estimate population density, visual counts were performed by
transect and quadrant. Activity patterns were evaluated in the transects. The damage was
assessed according to the cause and the tree species in 12 plots of $400 \mathrm{~m}^{2}$ and 13 points on a
path chosen at random. We found relatively high population densities, an average of 5.9 to 6.5
inds. / ha according to the methods of transect and quadrant, respectively. With these data, we
estimate between 254 and 270 squirrels in the park. They have two activity peaks, one in the 
morning and one before sunset. Just over half (52\%) of trees sampled (346) had some type of damage and of these $80 \%$ were damaged by vandalism and pruning, and the remaining $20 \%$ by squirrels. These results show that it is important that campaigns be conducted to avoid the squirrels food supplement and / or other species and that a training program to make a proper pruning of trees and thus prevent further damage.

Key words: Squirrel, population density, activity patterns, Viveros de Coyoacan, Distrito Federal.

\section{INTRODUCCIÓN}

Se espera que los paisajes naturales, en la continuación del siglo XXI, sigan con su proceso de transformación sin precedente iniciado hace más de 200 años, convirtiéndose en paisajes rurales y urbanos. Los manejadores y científicos de fauna silvestre intentan prepararse para estos cambios; sin embargo, es insuficiente la literatura disponible para fauna en áreas urbanas (Vandruff et al., 1996; Wolch et al., 1995). Las áreas urbanas han sido definidas como sitios de población humana relativamente densa o muy densa, donde la mayor parte del uso del suelo es dedicada a edificios, parques, concreto, terrenos baldíos y otros usos del suelo no agropecuarios (Adams et al., 2006).

Algunas poblaciones de fauna, como venados (Odocoileus spp.), ardillas (Sciurus spp.) y gansos (Branta spp.) han podido adaptarse a paisajes dominados por el humano, modificando parte de sus parámetros ecológicos básicos por un proceso definido como "síndrome de la fauna silvestre urbana" (Adams et al., 2005). Este proceso, que por simplificación ha sido llamado "síndrome de la urbanización", conduce a cambios en el tamaño poblacional, la estructura de sexos y edades, la supervivencia, el comportamiento y el uso del hábitat (Gliwicz et al., 1994)

Este conjunto de características ha sido descrita en las poblaciones urbanas de ardillas grises (Sciurus carolinensis Flyger, 1970), palomas silvestres (Columba livia - Cooke, 1980), coyotes (Canis latrans - Shargo, 1988), ratones de campo (Apodemus agrarius - Gliwicz et al., 1994), mirlos (Turdus merula - Gliwicz et al., 1994), mapaches (Procyon lotor Smith y Engeman, 2002) y serpientes de agua (Nerodia sipedon - Burger, 2001).

En México, se tiene conocimiento que existen ardillas arborícolas nativas e introducidas, en los parques y jardines de las ciudades de Oaxaca (Sciurus aureogaster), Mérida (S. yucatanicus), Tuxtla Gutiérrez (S. variegatoides y $S$. aureogaster), y del Distrito Federal ( $S$. aureogaster). Además, se ha documentado la introducción de dos poblaciones en Baja California Norte (Huey, 1964), aunque el destino de estas poblaciones permanece incierto (Koprowski et al., 2006).

Aunque la ardilla gris es una especie nativa de la Cuenca de México, ha sido 
introducida en parques urbanos de la Ciudad de México. Tal es el caso de los Viveros de Coyoacán, el cual fue creado para proveer de árboles a la ciudad. Los trabajadores del parque reportan daños por descortezamiento en los árboles y una disminución en la producción de semillas y plántulas; además, se piensa que la población de ardillas ha aumentado aunque no se ha vuelto a estimar el tamaño de la población (Cabrera, S. Administradora de los Viveros de Coyoacán de 1998-1999, com. pers.). Sin embargo, no existen estudios sistematizados que cuantifiquen el impacto de las ardillas en la vegetación del parque.

En este estudio nos planteamos los siguientes objetivos: 1) Estimar la densidad de la ardilla gris mexicana utilizando métodos visuales. 2) Analizar el patrón de actividad de las ardillas a lo largo de un año; y 3) Describir y cuantificar los daños ocasionados por las ardillas en los árboles de los Viveros de Coyoacán, contrastándolos con los daños ocasionados por otros agentes.

\section{MÉTODOS}

\section{Área de estudio}

El Parque "Los Viveros de Coyoacán" (coordenadas $19^{\circ} 26^{\prime} \mathrm{N}$ y 98²'W), con una altitud de 2,273 msnm, fue establecido en 1907, y es el primer vivero forestal del país. Es una de las áreas verdes más importantes del sur de la Ciudad de México y es un sitio de esparcimiento para la población, con una afluencia de más de 2,500 visitantes diariamente
(SEMARNAP, 1997). El objetivo principal de los Viveros es la producción de árboles y plantas de ornato, con más demanda y árboles de tipo urbano que han contribuido de manera considerable a la reforestación de la Ciudad de México. La producción anual promedio es de 500,000 árboles, principalmente de trueno (Ligustrum lucidum), cedro blanco (Cupressus sp), ciprés (Cupressus sp), fresno (Fraxinus udhei), pinos (Pinus sp.), encinos (Quercus sp.), ciruelo rojo (Prunus sp.) y liquidámbar ((Liquidambar styracifluaSEMARNAP, 1997). Cuenta con una superficie aproximada de 43 ha. y se ubica en el centro de la delegación Coyoacán (Figura 1).

Los árboles que se encuentran delimitando los lotes en toda la zona perimetral de los Viveros se plantaron en su gran mayoría entre 1920-1940, en la actualidad, son los de mayor altura. El estrato arbóreo es el de mayor representación en este lugar, quedando en segundo término el estrato arbustivo. Las herbáceas crecen de manera silvestre, teniendo poco valor visual o paisajístico. Las especies vegetales existentes en el parque son tanto de origen nacional como extranjeras y fueron introducidas cuando el parque fue creado en 1907. Entre las especies arbóreas más comunes se encuentran el encino, fresno liquidámbar, casuarina (Casuarina equisetifolia), aile (Alnus jorullensis), eucalipto (Eucaliptus sp.), trueno, pino, cedro, castaño (Castaneo sativa), ahuehuete (Taxodium mucrontum), pera (Pyrus communis), tejocote (Crataegus mexicana), mandarina (Citrus deliciosa) y guayaba 


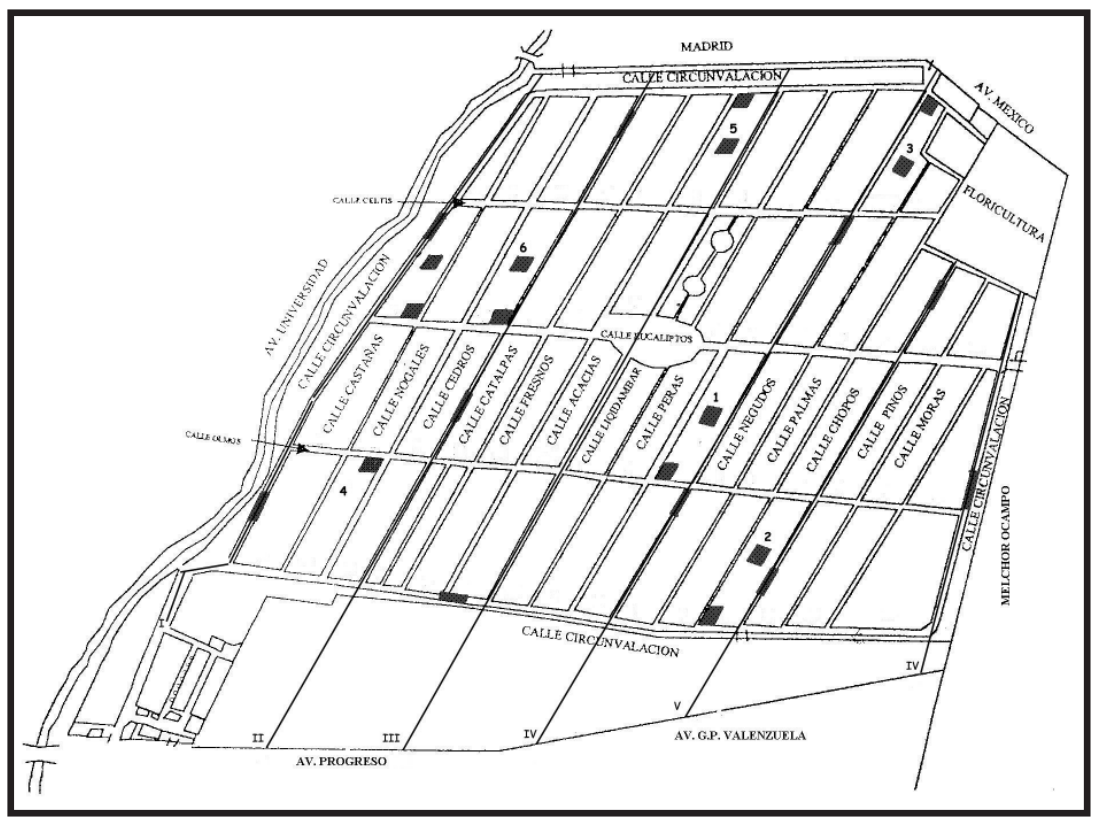

Figura 1. Mapa de los Viveros de Coyoacán, ubicación (transectos y cuadrantes), colindancias y tipo de vegetación por lote. Transecto/calle: I (C. U.) 601 m; II (Cedros) 600m; III (Acacias) 665m; IV (Jacarandas) 592m; V (Chopos) 392m; VI (C. M. O.) $339 \mathrm{~m}$. Lotes/árbol predominante: 1/Trueno, 4,480 $\mathrm{m}^{2}$; 2/Arboretum, 4,725m²; $3 /$ Liquidambar, 5,202m²; 4/Producción, 4,114m²; 5/Cipres, 4,235m² 6/Fresno, 4,092m². 
(Psidium guajava). Los arbustos más comunes son los truenos (Ligustrum lucidum y japonicum).

La capa superficial del suelo es de relleno con tierra lama extraída del río Magdalena, que es la única corriente superficial del parque. Este afluente baja desde la parte alta de la delegación Magdalena Contreras y se conecta con el río Churubusco el cual está entubado (SEMARNAP-CICEANA, 1997).

La estructura natural que tenía el terreno era sencilla, pedregoso y sin horizontes formados, ocasionó que al ser rellenado contara con un buen drenaje. El suelo cuenta con aproximadamente unos 2.5 o 3 metros de profundidad.

En el andador perimetral Circunvalación, el suelo está compactado debido al paso constante de usuarios.

La fauna del parque se encuentra representada principalmente por roedores (ardillas y ratas) y aves (palomas, cernícalos, gorriones, etc.). También se pueden encontrar gatos domésticos (obs. directa).

El tipo de clima de la zona es $c(W o)(w) b\left(i^{\prime}\right) g$, templado (húmedosubhúmedo, con lluvias en verano), con una temperatura media anual entre $12^{\circ} \mathrm{y}$ los $18^{\circ} \mathrm{C}$. La temperatura del mes más frío varía entre $-3^{\circ}$ y $18^{\circ} \mathrm{C}$ y la del mes más cálido entre $6.5^{\circ}$ y $22^{\circ} \mathrm{C}$. Tiene una oscilación térmica entre $5^{\circ}$ y $7^{\circ} \mathrm{C}$. La estación más seca es el invierno, presentando una temporada de sequía más corta en la primavera. El mes más frío se da entre diciembre y enero con una temperatura mínima entre los $12^{\circ} \mathrm{C}$ y $7^{\circ} \mathrm{C}$. Las lluvias se presentan en verano, teniendo un promedio anual de $596.5 \mathrm{~mm}$. (Datos obtenidos de la estación de monitoreo del Distrito Federal ubicada con el número 020 en la Facultad de Ingeniería de la UNAM)

\section{Densidad Poblacional}

Los conteos para estimar la densidad poblacional se llevaron a cabo mensualmente, de febrero de 1999 hasta marzo de 2000. Para estimar la densidad poblacional se utilizaron dos métodos, el método por transecto lineal y por cuadrantes.

De manera sistemática se escogieron 6 de 15 transectos potenciales con dirección suroeste-noreste, sobre las calles del parque (Figura 1). La longitud de los transectos fue igual al tamaño de las calles (Figura 1). Los 6 transectos escogidos tuvieron una longitud promedio de $531.6 m( \pm 132.36)$.

Cada transecto fue recorrido tres veces por mes durante el periodo de muestreo. Para evitar sesgos causados por los patrones de actividad de las ardillas, se seleccionó al azar la hora de los recorridos, ya sea por la mañana (9:00 a 10:00) ó por la tarde (16:00 a17:00 hrs), considerando que en un estudio previo las ardillas presentaron un máximo de actividad en estas horas (Ceballos et al., 1994). La velocidad fue de uno a dos $\mathrm{km} / \mathrm{h}$ aproximadamente (Mandujano, 1997). Se registró el número de ardillas observadas dentro de una franja de $20 \mathrm{~m}$ (considerando los $10 \mathrm{~m}$ a cada lado del centro del transecto), asumiendo que dentro de esta franja la probabilidad de detección era igual a 1. Para el cálculo de la densidad se aplicó la fórmula: 


$$
\mathrm{D}=\mathrm{n} / 2 \mathrm{wL}
$$

Donde:

$n=$ número de ardillas observadas, $L=$ suma total del largo del transecto (que fue la suma de la longitud de todos los transectos), $\mathrm{y}$

$W=$ ancho del transecto (Mandujano, 1994; 1997) en este caso fue constante porque la probabilidad de detección fue la misma en todos los transectos.

Se escogieron de manera aleatoria seis lotes, correspondientes a los tipos de vegetación que presentan una mayor cobertura en los Viveros (más de 4,000 m² por cuadrante o lote; Figura 1). La separación mínima entre cada uno de los lotes fue de cuando menos $250 \mathrm{~m}$, la cual es el doble de la distancia máxima de desplazamiento que se ha observado para las ardillas en estudios preliminares y se usó con el fin de reducir la probabilidad de contar dos veces a una misma ardilla. Se contaron todas las ardillas que se observaron dentro de cada cuadrante y caminando en zigzag para abarcar toda el área del lote. Cada cuadrante fue contado tres veces por mes durante el año de muestreo para obtener una media mensual y una media anual.

Las estimaciones para ambos métodos se efectuaron dentro del mismo periodo de muestreo (cinco días cada mes). Cada día se contaron entre tres o cuatro transectos y tres o cuatro cuadrantes, de manera que al final se obtuvieron tres repeticiones para cada transecto y cada cuadrante por periodo. El orden de muestreo se determinó de forma aleatoria al inicio del periodo de muestreo tomando en consideración cada uno de los métodos (transecto y/o cuadrante).

El número promedio de ardillas por mes para cada sitio por cada método (cuadrante y transecto) se utilizó para estimar la densidad mensual promedio y posteriormente obtener el promedio anual. Los valores de densidad obtenidos por cada método se compararon por medio de una prueba de $t$ (Programa SPSS).

\section{Patrones de actividad}

Con el fin de identificar variaciones en el número de ardillas observadas a lo largo del día, también en los horarios de mayor actividad, se recorrieron los seis transectos una vez por mes registrando el número de ardillas en diferentes periodos del día (1= 9:00-10:30, $2=11: 15-12: 45,3=13: 30-15: 00 y$ $4=15: 45-16: 00)$. Los resultados fueron comparados por medio de un ANOVA de una sola vía, para determinar si existían diferencias entre los periodos.

\section{Daño por descortezamiento}

Para estimar el daño por descortezamiento causado por las ardillas, se ubicaron al azar dos parcelas de 20 x 20m (400 m²) dentro de los lotes seleccionados para estimar la densidad de ardillas, una en el centro del lote y la otra en uno de los extremos (Figura 1). Se incluyo además un lote de casuarina que presentaba muchos árboles dañados, dando un total de 13 parcelas. Al inicio del estudio, en cada parcela, se revisaron todos los árboles marcando aquellos que presentaban daño por descortezamiento 
y los que no lo presentaban; de estos, se escogieron al azar 12 árboles con daño y 12 sin daño.

Sobre los 6 transectos de conteo de ardillas se escogieron 12 puntos al azar; en cada uno de ellos se tomaron 9 árboles descortezados y 9 árboles sin descortezar. Lo anterior con el fin de comparar la extensión del daño entre los árboles de los cuadrantes de en medio, los árboles de los cuadrantes de la periferia y los árboles de los transectos (Figura 1).

Para cada árbol elegido tanto de los lotes como de los puntos se registraron las siguientes características: especie, diámetro a la altura del pecho (DAP), etapa de desarrollo (briznal, joven, maduro, senil o muerto) y altura total. La superficie del tronco principal, se calculó mediante la fórmula del área total para un cilindro:

$$
A t=2 r h+2 r^{2}
$$

donde:

r: el radio del árbol a una altura de $1.50 \mathrm{~m}$ h: la altura total del árbol.

Para los árboles dañados se determinó: tipo de daño y superficie dañada. El tipo de daño se estableció con base en la causa y la antigüedad del daño: a) tipo de daño: Ardilla/Herida (vandalismo)/Poda. b) antigüedad: Reciente/Antiguo.

\section{RESULTADOS}

\section{Densidad poblacional de ardillas}

La densidad promedio anual de ardillas obtenida a partir de los transectos fue de 5.9 ardillas/ha. El mes en que se obtuvo la mayor densidad promedio fue en marzo de 1999, con 8.5 ardillas/ha y el de menor densidad fue enero del 2000, con 4.8 ardillas/ha, pero no hubo diferencias significativas entre ellos (Figura 2).

La densidad anual promedio de ardillas (ardillas/ha) obtenida a partir de los cuadrantes fue de 6.5 ardillas/ha. La densidad mensual estimada con este método es muy variable (Figura 2); por ejemplo, el mes en el que se obtuvo la mayor densidad promedio fue abril con casi 11 ardillas/ha y el mes que tuvo menos fue febrero con menos de 4 ardillas/ ha (Figura 2).

Se encontró que la densidad promedio por cuadrante no es significativamente diferente a la de transectos (6.5 vs 5.9 respectivamente; $t=-981$; gl 142; $p=0.3$ ).

\section{Cuadrantes y tipo de vegetación}

Se encontraron diferencias significativas en la abundancia de las ardillas entre cuadrantes o tipos de vegetación (ANOVA $F_{5,142},=10.34 p<0.001$ ). Los análisis post hoc (Prueba de Tukey) mostraron que los cuadrantes dominados por fresnos y truenos (cuadrantes 6 y 1 ) presentaron densidades significativamente mayores (Figura 2) con respecto a los cuadrantes 2, 3 y 4 (arboretum, liquidambar y producción, respectivamente). En general, también se encuentra diferencias significativas en la presencia de las ardillas del mes de abril con respecto al mes de enero y noviembre. 


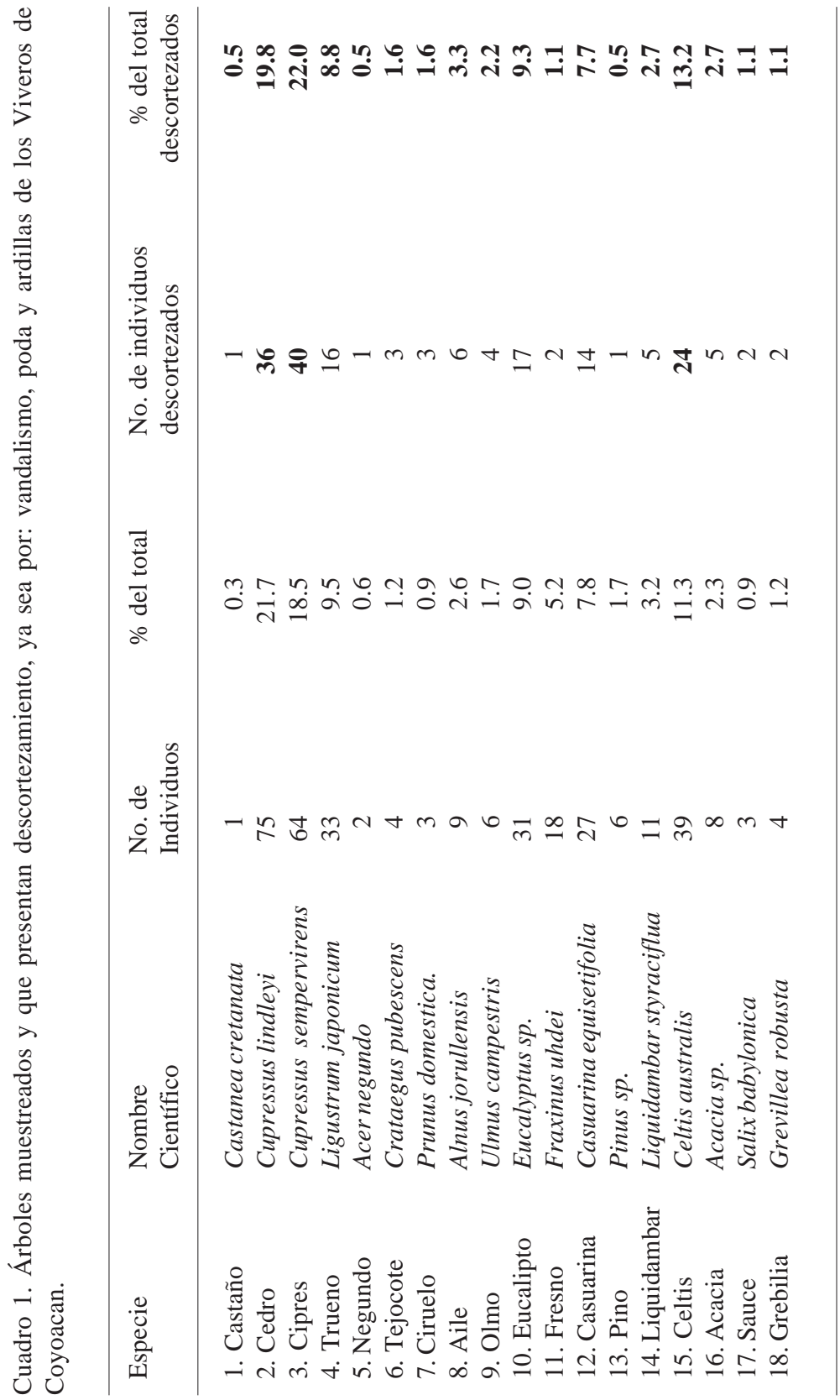




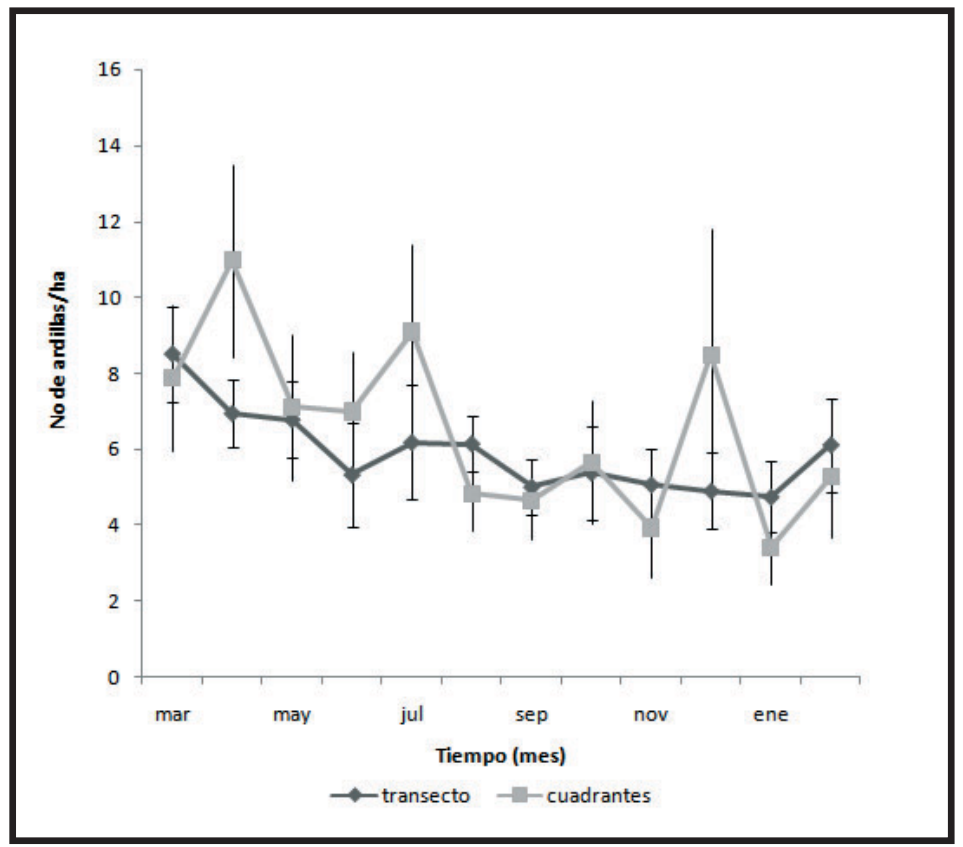

Figura 2. Abundancia (ardillas/ha.) por cada mes en los transectos y cuadrantes de los Viveros de Coyoacán.

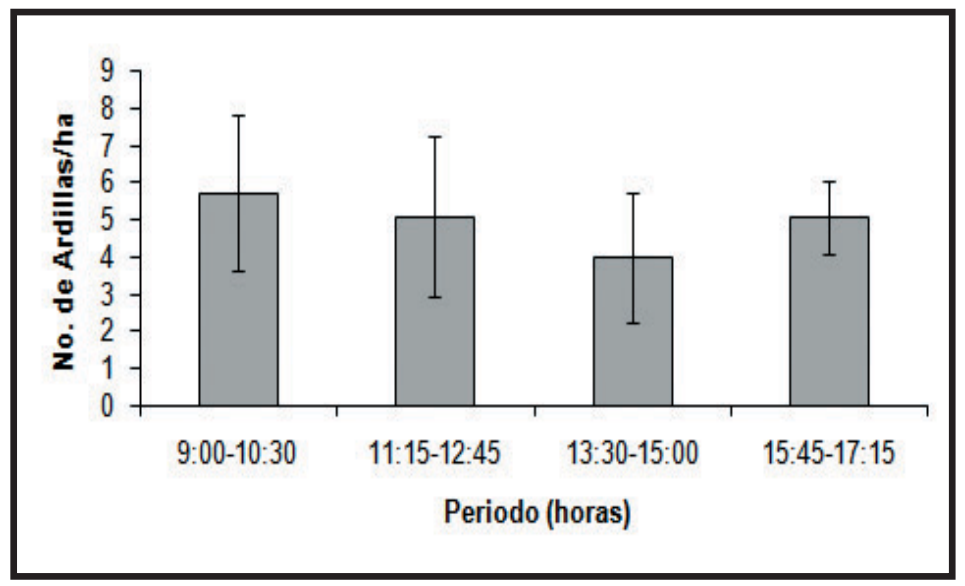

Figura 3. Actividad de las ardillas de los Viveros de Coyoacán por periodos de tiempo. 


\section{Patrones de actividad}

Los resultados de los conteos realizados en los cuatro periodos del día, nos muestran que en el periodo 1 (9:00 a 10:30) se observaron más ardillas, a diferencia del periodo 3 (13:30 a 15:00) que se observaron menos (Figura 3) y no se encontraron diferencias significativas entre los periodos (ANOVA $F_{3,11}$, $=1.86$ $p>0.05$ ).

\section{Daño por descortezamiento Especies de árboles}

El número total de árboles muestreados por ambos métodos (por puntos y lotes) fue de 346; la especie que tuvo un mayor número de individuos muestreados fue el cedro $(n)=75,21.7 \%$ ) seguida por el ciprés $(n=64,18.5 \%)$ y el celtis $(n=39,11.3 \%)$. Estas tres especies representaron más de la mitad (51.4\%) de las especies muestreadas; el resto (48.6\%) se reparte entre otras 16 especies (Cuadro 1).

Descortezamiento.-182 de 346 árboles muestreados presentaron uno o más de los tres tipos de descortezamiento (por ardilla, por herida o por poda). El resto no presentan ningún tipo de daño por descortezamiento (Cuadro 1). La mayoría de los árboles descortezados presentan heridas (122), 84 individuos por poda y sólo51 individuos muestran descortezamiento por ardillas. La suma de estos números es mayor al total de árboles revisados ya que algunos árboles presentaron más de un tipo de daño. La gran mayoría de los árboles (117) presentan un solo tipo de daño (64.3\%), mientras que 55 individuos presenta dos tipos de daños (30.2\%) y sólo 10 presentan tres tipos de daño (5.5\%).

De los 182 individuos descortezados, el ciprés fue la especie que presentó un mayor número de individuos 40 (22\%). En esta especie el tipo de descortezamiento que predominó fue por herida, con $86.09 \%$; seguido por poda, con $7.32 \%$, y por último por ardilla, con $6.59 \%$.

El cedro ocupó el segundo lugar, 34 individuos dañados, representando el $19.8 \%$ del total de árboles descortezados, siendo además la especie que tuvo un mayor número de individuos en toda la muestra (21.7\%). De los daños para esta especie, 28 (77.8\%) individuos fueron dañados por herida y por poda 17 (47.2\%), y sólo 3 presentaron daño por ardilla.

El celtis ocupó el tercer lugar con el 13.2\% (24 individuos) del total de árboles dañados. Para esta especie el tipo de descortezamiento que predominó fue por herida, con 83.3\% (20 individuos). Para las 16 especies restantes hubo menos de 17 individuos descortezados (Cuadro 1).

La especie que presentó mayor extensión de descortezamiento por ardilla fue el ciruelo con $256.78 \mathrm{~cm}^{2}$, seguido de la casuarina con $85.77 \mathrm{~cm}^{2}$ y el olmo con $60.96 \mathrm{~cm}^{2}$.

\section{Edad de los árboles}

La mayoría de los árboles fueron individuos maduros ( $n=264,76.3 \%$ ); seguido por los individuos seniles ( $\mathrm{n}=50,14.45 \%)$ y por los individuos juveniles $(n=22,6.36 \%)$ y finalmente, los individuos muertos ( $\mathrm{n}=10$, $2.89 \%)$. 
Cabe mencionar que no hubo ningún individuo en la etapa briznal.

\section{DISCUSIÓN}

El llamado "síndrome de urbanización" (Warren et al., 2006), se encuentra presente en las ardillas grises mexicanas (Sciurus aureogaster) de los Viveros de Coyoacán, ya que encontramos densidades poblacionales relativamente altas (en promedio 5.9 a 6.5/ha), en comparación con las estimadas en ambientes silvestres en México para esta y otras especies de ardilla. Por ejemplo en Huatusco, Veracruz, no se registró la presencia de ardilla gris en fragmentos de bosque tropical perennifolio mezclados con café (Tlapaya y Gallina, 2010), mientras que en un bosque tropical caducifolio y perennifolio del estado de Jalisco se reportaron densidades de 0.03 a 1.3/ha (Sciurus deppei; Curiel, 1992; Mandujano, 1997). En una zona cafetalera en Veracruz, México, sólo se indica que $S$. aureogaster es muy abundante (Tlapaya y Gallina, 2010).

Comparando nuestras estimaciones con las de otras especies de ardillas en Estados Unidos, se han reportado hasta 3 inds./ha en ambientes forestales ( $S$. carolinensis - Teaford, 1986). Nuestras estimaciones se encuentran dentro de las densidades estimadas en otros ambientes urbanos, las cuales van de 1 a 16 indiv/ha (S. carolinensis - Flyger, 1970; 1974; Thompson, 1978; Parker y Nillon, 2008). Sin embargo, nuestras estimaciones están muy por debajo de las estimadas en el "Lafayette Park", en Washington, D. C., en donde se reportan de 22.8 a 51.5 indiv/ ha (Maski et al., 1980; Parker y Nillon, 2008)

Las densidades estimadas por los dos métodos utilizados para la estimación poblacional de las ardillas son muy similares y no presentaron diferencias significativas.

Encontramos que el método de cuadrantes tiene una mayor variación (Figura 2), por lo tanto, este es muy dependiente de la estructura y estratificación del hábitat.

Se ha sugerido que para realizar un censo más confiable de ardillas se requiere de una combinación de métodos (Bouffard y Hein, 1978). Nuestros resultados indican que los métodos dependerán de la heterogeneidad/homogeneidad existente en cada sitio, por lo que uno u otro método puede ser utilizado dependiendo de los objetivos del estudio. Aunque en ambos métodos se invierte el mismo tiempo y el mismo esfuerzo, el método de los transectos parece ser el más adecuado para estimar la densidad de ardillas, sobre todo en ambientes heterogéneos.

\section{Patrones de actividad}

Los dos picos de actividad registrados en este trabajo para la ardilla gris mexicana (Sciurus auregaster), uno de 9:00 a 10:30 a.m. y otro de 13:30 a 15:00 hrs., son congruentes con los registrados para otras ardillas arborícolas (S. carolinensis - Thompson, 1977; S. niger - Adams, 1984; Geeslin, 1970; Hicks, 1949; S. vulgaris - Wauters et al., 1995).

Las ardillas grises de los Viveros de Coyoacán reducen su actividad en las horas más cálidas del día, lo cual ocurrió 
en casi todas las temporadas del año. Sin embargo, la mayoría de las especies de ardillas de Norteamérica y Europa, presentan un cambio en su actividad, presentándose un cambio unimodal, en el cual este aumenta conforme a las horas más calidas del día, sobre todo en la temporada invernal (Wauters et al., 1992).

Se ha especulado que la variación en la longitud del día y la temperatura explica el $90 \%$ de la variación en el tiempo de actividad. Esto es generalmente aceptado, ya que a bajas temperaturas, las ardillas descansan en su nido, en donde la temperatura en los inviernos fríos puede ser de 20 a $30^{\circ} \mathrm{C}$ arriba de la temperatura ambiental (Pulliainen, 1973). Esta importante respuesta conductual, resulta de un decremento de los costos energéticos de termorregulación (Wauters y Dhondt, 1987). Dado que las ardillas de los Viveros viven en un clima templado (temperatura media anual entre $12^{\circ}$ y $18^{\circ} \mathrm{C}$ ) y que la fluctuación del periodo del día es de 11 a 14 horas de radiación solar, las horas de menor actividad suelen ser aquellas de mayor calor, entre las 13:30 y 15:00 hrs.

\section{Daño descortezamiento}

El descortezamiento de los árboles es otro daño importante ocasionado por las ardillas, tanto en zonas urbanas como en plantaciones (Genovesi, 1998; Gurnell, 1989; Irving y Beer, 1963; Kenward y Parish, 1986; Manski, et al., 1981), además promueve infecciones de hongos o insectos (Kenward, 1989), disminuyendo la producción de los árboles y causando pérdidas económicas. Es interesante mencionar, que sólo un poco más de la quinta parte de los árboles con daño en los Viveros ha sido afectada por las ardillas. La gran mayoría de los daños resulta básicamente de las malas prácticas y del vandalismo.

El mayor número de árboles dañados por descortezamiento provocado por las ardillas, en los Viveros, correspondió a especies como la casuarina (Casuarina equisetifolia), eucalipto (Eucalyptus sp.), ciruelo (Prunus domestica) y tejocote (Crataegus pubescens). En Inglaterra, por ejemplo, el descortezamiento ocasionado por las ardillas ocurre principalmente a mediados del verano, siendo las especies más vulnerables el sicomoro (Acer pseudoplatanatus), las hayas (Fagus sylvatica) y los encinos (Quercus spp. Kenward y Parish, 1986).

La extensión del daño por descortezamiento, se ha correlacionado con la cantidad de floema en los árboles y con la densidad de ardillas juveniles en el área. También se ha mencionado que el daño por descortezamiento no se encuentra directamente relacionado con la densidad de ardillas adultas en los lugares donde existen árboles juveniles, porque también puede ocurrir éste en lugares con árboles de mayor edad (13-14 años; Kenward, 1989).

\section{Implicaciones para la conservación}

Las altas densidades de las ardillas que se encuentran en los Viveros de Coyoacán, se pueden explicar por diversos factores entre los que destacan: la adición y 
disponibilidad de alimento suplementario antropogénico, ya sea de forma conciente o inconsciente (comederos para aves u otras especies), dentro del área de los Viveros o en sus cercanías. Aunque no se midió el potencial aporte de alimento que pueden dar los visitantes, si se observó grupos de hasta 10 ardillas recibiendo alimento por parte de los mismos, en otros estudios se ha observado que éste puede corresponder al 35\% de la dieta de las ardillas (Hadidian et al., 1988).

Fue relativamente común ver movimientos de algunos individuos fuera de los Viveros hacia áreas cercanas como parques, camellones y en los árboles de algunas casas. Es probable que algunos de estos individuos vivan en la periferia del parque o bien se desplacen fuera del mismo por un proceso de dispersión. Patrones conductuales similares han sido observados en los que las ardillas pueden salir del parque, en donde se han seguido individuos por 20 minutos alejándose a una distancia de $0.48 \mathrm{~km}$ (Manski, 1981).

La densidad y el estado aparente de las ardillas de los Viveros de Coyoacán, indican que la población se encuentra en buenas condiciones. Sin embargo, existen varias acciones que sería conveniente emprender para mantener a la población en buen estado. Por ejemplo, recomendamos realizar estudios demográficos que nos permitan evaluar si la población se encuentra estable, si está aumentado o está decreciendo.

Es una población de aproximadamente 260 ardillas, la cual está relativamente aislada, por lo que es necesario evaluar la estructura genética de la población para conocer el riesgo de depresión por endogamia. También es necesario evaluar el estado de salud de las ardillas y observar la relativa abundancia de las especies de fauna nociva (ratas y ratones). Asimismo, será necesaria la captura de algunos ejemplares, con la finalidad de hacer exámenes sanguíneos y tejidos, para evaluar parásitos y enfermedades. Esto permitirá conocer qué enfermedades se encuentran en las ardillas y en la fauna nociva ó nativa (ratas y ratones), que potencialmente pudieran ser trasmitidas al hombre de forma directa por las ardillas.

Es necesario realizar periódicamente el corte de malezas, y el amontonamiento de desperdicios, los cuales puedan se utilizados por ratas y/o gatos como madrigueras y evitar permanentemente la entrada de perros y gatos, eliminando aquellos que se han establecido dentro de los Viveros.

Es importante seguir instrumentado programas de educación a los visitantes de los viveros en los cuales se indiquen las medidas de manejo instrumentadas, además de la importancia de no suplementar alimento a las ardillas y/o a otras especies.

\section{AGRADECIMIENTOS}

Al Dr. Ceballos y al laboratorio de Ecología y Conservación de Fauna Silvestre, del Instituto de Ecología UNAM, por su apoyo y permitir realizar la presente investigación. A las autoridades de los Viveros de Coyoacán por las facilidades prestadas. A Yolanda Domínguez por su revisión y a los dos revisores 
anónimos que con sus comentarios enriquecieron el trabajo.

\section{LITERATURACITADA}

Adams, C.E. 1984. Diversity in fox squirrel spatial relationships and activity rhythms. Texas Journal of Science, 36:197-203.

Adams, L.W., L.W. Van Druff, y M. Luniak. 2005. Managing urban habitats and wildlife. Pp. 714-739, en: Techniques for wildlife investigations and management. (C.E. Braun, ed.) Allen Press, Inc., Lawrence, Kansas, USA.

Adams, C.E., K.J. Lindsey, y S.J. Ash. 2006. Urban wildlife management. Taylor and Francis, Boca Raton, Florida, USA.

Bouffard, S.H. y D. Hein. 1978. Census methods for eastern gray squirrels. Journal Wildlife Management, 42:550557.

Burger J. 2001. The behavioral response of basking Northern water (Nerodia sipedon) and Eastern garter (Thamnophis sirtalis) snakes to pedestrians in a New Jersey park. Urban Ecosystem, 5:119-129

Ceballos, G., Pacheco, J. y C. Chávez. 1994. Diagnostico y manejo relativo a la población de ardillas que existen en los Viveros de Coyoacán. Informe Técnico. SARH.

Cooke A.S. 1980.Observations on how close certain passerine species will tolerate an approaching human in rural and suburban areas. Biological Conservation, 18:85-88.

Flyger, V.F.1970. Urban gray squirrelsproblems, management, and comparisons with forest populations. Trans Northeast Fish Wildlife Conference, 27:107-113

Flyger, V.F. 1974. Tree squirrels in urban environments. Pp. 121-123. en: Wildlife in an urbanizing environment. (J.H. Noyes y D.R. Progulski, eds.)
Holdsworth Natural Recourse Center Series 28, University of Massachusetts, Amherst, Massachusetts, USA

Geeslin, H.G. 1970. A radio-tracking study of home range, movements, and habitat uses of the fox squirrel (Sciurus niger) in east Texas. M.S.Thesis, Texas AyM University, College Station, Texas, USA.

Genovesi, P. 1998. Italy's gray squirrels: Action vs. opposition. World Conservation, 1:25-26.

Gliwicz, J., J. Goszczynski, y M. Luniak. 1994. Characteristic features of animal populations under synurbanization-the case of the blackbird and striped field mouse. Memorabilia Zoologica, 49:237244.

Gurnell, J. 1989. Demographic implications for the control of gray squirrels. Pp. 131143, en: Mammals as pests. (R.J. Putman, ed.). Chapman and Hall. New York, E.U.A.

Hadidian J., Manski D., Flyger V., Cox C., y G. Hodge. 1988. Urban gray squirrel damage and population management: a case history. Proceedings of the Third Eastern Wildlife Damage Control Conference, 219-227

Hicks, E.A. 1949. Ecological factors affecting the activity of the western fox squirrel, (Sciurus niger rufiventer) (Geoffroy). Ecological Monographs, 19:287-302.

Huey, L.M. 1964. The mammals of Baja California, Mexico. Transactions of the San Diego Society of Natural History, 13:85-168.

Irving, F.D. y J.R. Beer. 1963. A six-year record of sugar maple bark-stripping by gray squirrels in a Minnesota oak-maple stand. Journal Forestry, 61:508-511.

Kenward, R.E. y T. Parish. 1986. Barkstripping by gray squirrels (Sciurus carolinensis). Journal Zoological London, 210:473-481. 
Kenward, R.E. 1989. Bark-stripping by gray squirrels in Britain and North America: why does the damage differ? Pp. 144-154, en: Mammals as pests. (R.J. Putman, ed.) Chapman and Hall. New York, E.U.A.

Koprowski, J.L., N. Ramos, B.S. Pasch, YC.A. Zugmeyer. 2006. Observations on the ecology of the endemic Mearns's squirrel (Tamiasciurus mearnsi). The Southwestern Naturalist, 51:426-430

Mandujano, S. 1997. Densidad poblacional de la ardilla gris del Pacífico (Sciurus colliaei) en un bosque tropical caducifolio de Jalisco. Revista Mexicana de Mastozoología, 2:90-96.

Mandujano, S. 1994. Conceptos generales del Método de Conteo de animales en transectos. Ciencia, 45:203-211.

Manski, D.A., L.W. Van Druff y V. Flyger. 1981. Activities of gray squirrels and people in Dowtown Washingtong, D.C. Park: Management Implications. Trans. North. American Wildlife Management, 46:439-454.

Parker, T. y C. H. Nilon. 2008. Gray squirrel density habitat suitability and behavior in urban parks. Urban Ecosystem, 11:243255

Pulliainen, E. 1973. Winter ecology of the red squirrel in northeastern Lapland. Annales Zoologici Fennici,10: 437-449.

SEMARNAP, 1997. Folleto "Viveros de Coyoacán". Gobierno de México.

SEMARNAP-CICEANA, 1997. Plan rector para el Centro de Cultura Ambiental de los Viveros de Coyoacán. SEMARNAP, México, D.F.

Shargo, E.S. 1988. Home range, movements, and activity patterns of coyotes (Canis latrans) in Los Angeles suburbs. Ph.D. Dissertation, University of California, Los Angeles. E.U.A.

Smith H. y R., Engeman. 2002. An extraordinary raccoon, Procyon lotor, density at an urban park. Canadian Field Naturalist, 116:636-639.

Thompson, D. C. 1977. Diurnal and seasonal activity of the grey squirrel (Sciurus carolinensis). Canadian Journal of Zoology, 55:1185-1189.

Thompson, D.C. 1978. Regulation of a northern gray squirrel (Sciurus carolinensis) population. Ecology, 59:708-715.

Tlapaya L. y S. Gallina. 2010. Cacería de mamíferos medianos en cafetales del centro de Veracruz, México. Acta Zoológica Mexicana (n.s.), 26:259-277.

Vandruff, L.W., E.G. Bolen, y G.J., San Julian. 1996. Management of urban wildlife. Pp. 507-530, en: Research and management techniques for wildlife and habitats. (T.A. Bookhout, ed.) Allen Press, Lawrence, Kansas, U.S.A.

Warren P., Tripler, C., Bolger, D., Faeth, S., Huntly, N., Lepczyk, C., Meyer, J., Parker, T., Shochat, E., y J., Walker. 2006. Urban food webs: predators, prey, and the people who feed them. Bulletin of the Ecological Society of America, 87:387-393

Wauters, L., Swinnen, C. y Dhondt, A. A. 1992 Activity budget and foraging behaviour of red squirrels (Sciurus vulgaris, Linnaeus 1758) in coniferous and deciduous habitats. Journal of Zoology (London), 227:71-86.

Wauters, L.A., Suhonen, J. y A. A. Dhondt. 1995. Fitness consequences of hoarding behaviour in the Eurasian red squirrel. Proceedings Royal Society, London B, 262: 277- 281.

Wauters, L.A. y A.A. Dhondt 1987. Activity budget and foraging behaviour of the red squirrel (Sciurus vulgaris, Linnaeus, 1758) in a coniferous habitat. $Z$. Saugetierk, 52:341-353.

Wolch, J.R., K. West y T.E. Gaines. 1995. Trans-species urban theory. Environment 
and Planning D: Society and Space, 13:735-760. 